\title{
The use of enfuvirtide-based HAART regimens in HIV patients undergoing chemotherapy for lymphoma
} BA White*1, R Fox ${ }^{1}$, J Laird ${ }^{2}$, A Seaton ${ }^{1}$ and A MacConnachie ${ }^{1}$

\author{
Address: ${ }^{1}$ Department of Infectious Diseases, Brownlee Centre, Gartnavel General Hospital, Glasgow, UK and ${ }^{2}$ Haematology Department, \\ Gartnavel General Hospital, Glasgow, UK \\ * Corresponding author
}

from Ninth International Congress on Drug Therapy in HIV Infection

Glasgow, UK. 9-13 November 2008

Published: 10 November 2008

Journal of the International AIDS Society 2008, I I (SuppI I):P29| doi:|0.|I86/I758-2652-I I-SI-P29 I

This abstract is available from: http://www.jiasociety.org/content/I I/SI/P29 I

(C) 2008 White et al; licensee BioMed Central Ltd.

\section{Purpose of the study}

To evaluate the use of enfuvirtide-based HAART regimens in patients receiving chemotherapy for lymphoma, where the concomitant use of antineoplastic agents with PI- or NNRTI-based regimens is difficult due to P450 enzyme and p-glycoprotein induction and inhibition.

\section{Methods}

This was a retrospective analysis of five HIV patients treated with an enfuvirtide-based HAART regimen while receiving chemotherapy for lymphoma. Data were gathered from their case notes and information regarding potential drug interactions was obtained from the HIV specialist pharmacist and literature review.

\section{Summary of results}

The five patients studied were all male, median age of 30 yrs (range 28-42), with no significant co-morbidities. Median baseline HIV viral load (VL) was 90,000 copies/ $\mathrm{ml}$ (range 11,759-3,035,664). Patients 1 and 2 with Burkitts Lymphoma, and Patient 3 with HIV-assoc DLBCL of anus (stage 1E) were treated with CODOX-M/IVAC; Patient 4 had high grade $\mathrm{B}$ cell lymphoma and received $\mathrm{r}$ CHOP. Each of these patients were treated with tenofovir/ emtricitabine and enfuvirtide. Patient 5 had plasmablastic high grade B cell NHL of the pancreas, which recurred in the leg. He received ESHAP/Ifosphamide/Cytarabine and his HAART regimen was zidovudine/lamivudine/tenofovir with enfuvirtide. Initial virological response was excellent in four patients, with all having more than $2 \log$ drop in VL within the first 3-7 weeks of treatment. VL was undetectable within 12 weeks in Patients 1 and 2. Patient 4 initially did very well, followed by virological relapse. He subsequently admitted to poor compliance and resistance testing revealed lamivudine resistance. Patient 5 had an initial excellent virological response but the lymphoma progressed despite therapy and he died 8 weeks into treatment. Patient 3 had a $0.9 \log$ drop in VL at week 5 and undetectable VL at week 19 which was then sustained throughout treatment. Two patients developed severe mucositis due to methotrexate, but none of the patients required dose reductions in their chemotherapy regimens. Two patients developed injection site reactions with enfuvirtide but continued to tolerate it for the duration of the chemotherapy. No patients developed renal dysfunction.

\section{Conclusion}

An enfuvirtide-based HAART regimen is efficacious and useful in HIV patients undergoing chemotherapy for lymphoma due to its lack of drug interactions with the antineoplastic agents.

\section{References}

I. Antoniou T, Tseng A: Interactions between antiretrovirals and antineoplastic drug therapy. Clin Pharmacokinet 2005, 44 (2): III-145. 